\title{
Prospecção Tecnológica em Documentos de Patentes que Apontam a Fosfoetanolamina como Substância Associada ao Diagnóstico, à Prevenção ou ao Tratamento de Algum Tipo de Câncer
}

\author{
Technological Prospection in Patent Documents that appoint \\ Fosfoetanolamine as a Substance Associated with Diagnosis, Prevention \\ or Treatment of Any Type of Cancer
}

\author{
Tarcísio Magalhães Cerqueira ${ }^{1}$ \\ Marcelo Miguel da Silva ${ }^{2}$ \\ Nubia Moura Ribeiro ${ }^{3}$ \\ ${ }^{1}$ Universidade Estadual de Feira de Santana, Bahia, BA, Brasil \\ ${ }^{2}$ Instituto Federal da Bahia, Simões Filho, BA, Brasil \\ ${ }^{3}$ Instituto Federal da Bahia, Jequié, BA, Brasil
}

\begin{abstract}
Resumo
O presente trabalho mostra um estudo prospectivo realizado em bases de dados de documentos de patentes acerca de documentos que apresentam a fosfoetanolamina como substância associada ao diagnóstico, à prevenção ou ao tratamento de algum tipo de câncer. Esse tema teve grande repercussão nos anos de 2015 e de 2016 e ainda repercute nos dias atuais. Tudo isso, pelos rumores de que um químico brasileiro havia "descoberto" a cura de algumas das neoplasias malignas, por meio do emprego dessa substância, em uma forma sintética especial. As bases de dados utilizadas foram a do Instituto Nacional da Propriedade Industrial (INPI), do Escritório Europeu de Patentes (European Patent Office, ESPACENET) e a do software ORBIT ${ }^{\circledR}$. Os resultados da pesquisa demonstraram que depósitos de patentes com essa temática existem desde 1983, os Estados Unidos lideram o domínio dessa tecnologia, houve um pico de depósitos no ano de 2014 e os maiores titulares da tecnologia apontada são pessoas jurídicas.
\end{abstract}

Palavras-chave: Fosfoetanolamina. Patentes. Prospecção.

\begin{abstract}
The present work shows a prospective study carried out in databases of patent documents on documents that present phosphoethanolamine as a substance associated with the diagnosis, prevention or treatment of some type of cancer. This theme had great repercussion in the years 2015, 2016 and still has repercussions in the present day. All this, by the rumors that a Brazilian chemist had "discovered" the cure of some of the malignant neoplasms, through the use of this substance, in a special synthetic form. The databases used were the National Institute of Industrial Property (INPI), the European Patent Office (ESPACENET) and the ORBIT ${ }^{\circledR}$ software. The results of the research have shown that patent deposits with this theme have existed since 1983, the US leads the domain of this technology, there was a peak of deposits in the year 2014 and the biggest holders of the technology pointed out are legal entities.
\end{abstract}

Keywords: Phosphoethanolamine. Patents. Prospection.

Área Tecnológica: Propriedade Intelectual. 


\section{Introdução}

A fosfoetanolamina, fórmula molecular C2H8NO4P é naturalmente produzida pelos mamíferos, o que inclui o homem. Sua produção acontece no retículo endoplasmático das células eucariontes, que contêm um núcleo e organelas protegidas por membranas. Tal substância é precursora da fosfatidilcolina e fosfatidiletanolamina, moléculas envolvidas na síntese de fosfolipídeos, uma classe de gorduras que são o principal constituinte das membranas celulares (PIVETTA, 2016).

A ligação da fosfoetanolamina com o câncer remonta a 1936, quando foi isolada, pela primeira vez, de tumores bovinos (PIVETTA, 2016). Os métodos para sua síntese e para a sua produção artificial já são conhecidos desde a década de 1950 e algumas variedades sintéticas, utilizadas como suplementos alimentares, são largamente comercializadas no exterior (ORSI, 2017).

Nos anos 2015 e 2016, em todo o Brasil, cresceu assustadoramente o interesse sobre o tema. No centro de toda a questão estava um químico aposentado da USP de São Carlos, Gilberto Chierice e a sua equipe. Eles alegavam ter desenvolvido um novo método para a produção da fosfoetanolamina sintética, com alto rendimento e baixo custo (PIVETTA, 2016). Além disso, essa equipe alegava também que o produto era detentor de propriedades especiais no combate a cinco tipos de câncer (ORSI, 2017).

Ao se espalhar a notícia de que aquela substância, produzida de forma simples, era portadora de propriedades únicas no combate ao câncer, conforme relataram os seus inventores, travou-se uma verdadeira batalha entre os mais diversos setores da sociedade brasileira.

De um lado, a favor da permissão da produção e utilização daquele composto, de forma indiscriminada, sem precisar passar por todas as etapas exigidas pelos órgãos fiscalizadores de medicamentos, estavam alguns setores da sociedade civil; alguns tribunais de justiça estaduais, os quais concederam liminares determinando a distribuição do medicamento em favor daqueles que os solicitaram; a maioria dos parlamentares do Congresso Nacional e a presidente em exercício no período, a qual sancionou, em caráter de urgência, a Lei n. 13.269/2016, que autorizava a produção, importação, prescrição, posse ou uso do composto farmacêutico, antes mesmo dos testes pré-clínicos e clínicos da ANVISA (ORSI, 2017).

De outro lado, existiam muitos críticos a toda essa dinâmica de priorização daquele composto e da forma como ela estava acontecendo, desrespeitando todas as etapas de segurança das pesquisas científicas. Tal conjunto era formado pelos próprios órgãos de fiscalização, como a ANVISA, uma grande parcela dos médicos e a quase totalidade da comunidade científica (ORSI, 2017).

No mês seguinte à publicação e à entrada em vigor da Lei n. 13.269/2016, o Plenário do Supremo Tribunal Federal (STF), por maioria de votos, deferiu medida liminar na Ação Direta de Inconstitucionalidade (ADI) 5501, proposta pela Associação Médica Brasileira (AMB), para suspender a eficácia da referida lei (STF, 2016).

Suspensa a eficácia da lei que liberava a produção, importação, prescrição, posse ou uso da fosfoetanolamina, antes mesmo dos testes da ANVISA, tornou-se necessário que o composto passasse pelo crivo da agência de vigilância sanitária. Iniciados os primeiros testes in vivo, os resultados divulgados eram de que a eficácia da substância no tratamento dos tipos de câncer mais comuns aos brasileiros estava muito aquém do que alegaram, categoricamente, os inventores do medicamento (ORSI, 2017). 
Contudo, os inventores contestaram a forma como foram realizados aqueles testes, apontando inúmeras falhas nos procedimentos. Diante dessas contestações, até o presente momento, há um impasse sobre a realização de novos testes com essa substância (ORSI, 2017).

Portanto, toda a polêmica em torno da fosfoetanolamina, apontada, por alguns, como composto revolucionário no tratamento de alguns tipos de câncer, não está superada.

Segundo Souza e Prado (2013, p. 194),

[...] as informações contidas em bancos de patentes são valiosas para a identificação de possíveis projetos ou a determinação de alteração do escopo de uma pesquisa em andamento, ou, ainda, para buscar novas soluções para as questões até o momento sem respostas.

A documentação de patentes é a mais completa entre as fontes de pesquisa. Estudos revelam que $70 \%$ das informações tecnológicas contidas nesses documentos não estão disponíveis em qualquer outro tipo de fonte de informação (INPI, 2016).

Entre as principais bases gratuitas de patentes, se destacam: INPI (Escritório Regional Brasileiro) e Espacenet (Escritório Europeu de Patentes). E das bases comerciais, o ORBIT® é um dos mais completos software com esSa função.

A base de patentes do INPI possibilita a busca de pedidos e de patentes concedidas depositados no Brasil, a partir de 1982. A sua atualização é semanal e permite busca básica, avançada e por número de patente. EsSa base reúne todas as áreas do conhecimento, permite pesquisas somente em português e possibilita a utilização recursos de pesquisa operadores booleanos (AND, OR e AND NOT) e caracteres de truncamento (*). A busca fornece somente dados bibliográficos dos pedidos e também apresenta o andamento dos processos, com os despachos já publicados (SOUZA; PRADO, 2013).

A base de patentes do Escritório Regional Europeu (ESPACENET) permite acesso a documentos do escritório europeu e de mais de 80 países, inclusive o Brasil. A sua atualização é semanal e permite busca rápida, avançada, por número, ECLA e pela CIP. A base também abrange todas as áreas do conhecimento e possibilita a utilização de operadores booleanos (AND, OR e NOT) e caracteres de truncamento (* para um número ilimitado de caracteres; ? para 0 ou um caractere), \# Um caractere exatamente). Os documentos estão disponíveis até 1970 e a base possui mais de 60 milhões de patentes (SOUZA; PRADO, 2013).

O Orbit Intelligence é um sistema de busca, seleção, análise e exportação de informações contidas em patentes. Produzido pela Questel Orbit, Inc., sua cobertura geográfica compreende publicações de quase uma centena de países e autoridades de patentes. Dados sobre titulares, inventores, textos, imagens, citações, tramitação e litígio são reunidos em duas bases de dados proprietárias: FamPat (famílias de patentes) FullPat (pedidos individuais). Possui amplas opções de buscas: Busca básica; Busca por menus; Busca por linhas de comando avançadas; Busca por citações; Busca por similaridade; Busca por famílias de patentes expandidas; dentre outras. E permite a exportação de dados em diversos formatos: .txt, .pdf, .rtf, .xls, .xlsx, .csv, .xml, texto, imagem, campos selecionados, hyperlinks permanentes (SUZUKI, 2017).

A justificativa para a escolha das bases de dados empregadas na pesquisa são as seguintes:

a) INPI: base de patente de escritório nacional; gratuita; geralmente, constitui o primeiro contato do(a) pesquisador(a). 
b)ESPACENET: base gratuita, com vasta abrangência e vários recursos, ferramentas e opções de pesquisa.

c) ORBIT $®$ : ferramenta comercial com desempenho muito superior às bases gratuitas, proporcionando a exportação e manuseio de dados em diversas extensões de arquivos, além de possuir uma maior quantidade de conectores e operadores de truncamento (GUERREIRO et al., 2017) e por conta da licença disponibilizada pela Axonal Consultoria Tecnológica aos mestrandos e docentes do Mestrado Profissional em Propriedade Intelectual e Transferência de Tecnologia para Inovação (PROFNIT, 2018).

O objetivo geral desta pesquisa foi fazer um mapeamento tecnológico inédito dos documentos de patentes que apontam a fosfoetanolamina como substância associada ao diagnóstico, à prevenção ou ao tratamento de alguns tipos de câncer, em três bases de dados distintas, de modo a comparar os resultados obtidos em cada base.

\section{Metodologia}

Trata-se de uma pesquisa exploratória, pois se restringiu a definir objetivos e a buscar mais informações sobre determinado assunto, também pretende obter uma nova percepção do assunto e descobrir novas ideias.

Como pesquisa do tipo quantitativa, ateve-se a expor, quantificar e discutir, por meio de gráficos, a quantidade de dados expressa por cada situação investigada, como, por exemplo, o número de documentos de patentes recuperados em relação aos seus principais depositantes, em relação aos seus inventores, em relação aos 20 últimos anos de depósito etc., nas três bases de dados utilizadas.

A estratégia de busca empregada nas bases INPI, ESPACENET e ORBIT $®$ fez uso de palavras-chave em detrimento dos Códigos Internacionais de Patentes (IPCs), uma vez que estavam bem definidos os termos pesquisados e os seus sinônimos. Tal estratégia permitiu se obter uma maior quantidade de resultados (Quadro 1).

A estratégia utilizada levou em consideração também as particularidades de cada base de dados em discussão, como a abrangência e os recursos disponíveis para a pesquisa. Os operadores booleanos utilizados foram o OR e o AND em todas as bases.

Quadro 1 - Estratégia de busca nas bases de dados

\begin{tabular}{|c|c|c|}
\hline INPI & ESPACENET & ORBIT® \\
\hline $\begin{array}{c}\text { Apenas no Título e apenas no Resumo: } \\
\text { Palavras-chave: } \\
\text { fosfoetanolamina or } \\
\text { fosfatidiletanolamina or fosfatidil } \\
\text { etanolamina or fosfatidilcolina and } \\
\text { câncer or neoplasia maligna }\end{array}$ & $\begin{array}{c}\text { Título ou Resumo } \\
\text { Palavras-chave: } \\
\text { (phosphoethanolamine or } \\
\text { phosphatidylethanolamine or } \\
\text { phosphatidylcholine) and (cancer } \\
\text { or "malignant neoplasm") }\end{array}$ & $\begin{array}{l}\text { Título ou Resumo } \\
\text { Palavras-chave: } \\
\text { (phosphoethanolamine or } \\
\text { phosphatidylethanolamine or } \\
\text { phosphatidylcholine) and (cancer } \\
\text { or "malignant neoplasm") }\end{array}$ \\
\hline
\end{tabular}

Fonte: Elaborado pelos autores deste artigo (2018) 
As buscas foram realizadas no final de setembro de 2018 e o período de tempo considerado na pesquisa, que mostra a evolução dos depósitos dos pedidos de patentes, foi de 1998-2018 (20 anos).

\section{Resultados e Discussão}

Os resultados obtidos com a prospecção foram agrupados por assunto e tiveram seus gráficos expressos de modo simultâneo para as três bases de dados pesquisadas, o que facilita a discussão e a comparação dos dados.

Primeiramente, foi exposta a quantidade de documentos de patentes recuperada em cada base de dados (Gráfico 1), fruto da estratégia de busca nela empregada, por palavras-chave, conforme disposto na metodologia do trabalho.

Foi verificado que as bases de dados do ESPACENET e do ORBIT $®$ apresentaram resultados próximos (88 e 102 patentes, respectivamente) e bem maiores do que o INPI (9 patentes), o que já era esperado, tendo em vista a maior abrangência daquelas bases internacionais.

Gráfico 1 - Quantidade de patentes recuperadas, dos últimos 20 anos, utilizando a fosfoetanolamina (OR fosfatidiletanolamina, OR fosfatidilcolina) AND câncer (OR neoplasia maligna) como resultado das buscas em cada base de dados

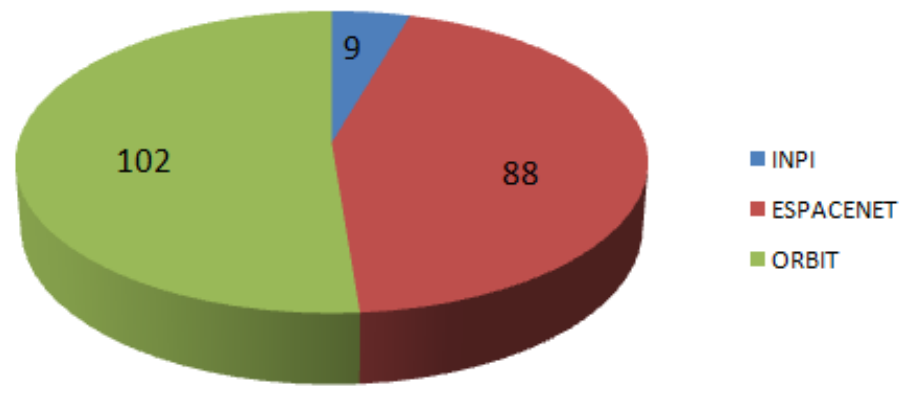

Fonte: Elaborado pelos autores deste artigo (2018)

No que se refere aos países de depósitos, na base de dados do INPI, 68,18\% das patentes depositadas são brasileiras ou têm no Brasil prioridade, $18,18 \%$ são americanas ou têm nos Estados Unidos prioridade, conforme demonstrado no Gráfico 2.

Gráfico 2 - Países de depósitos ou prioridade unionista - INPI

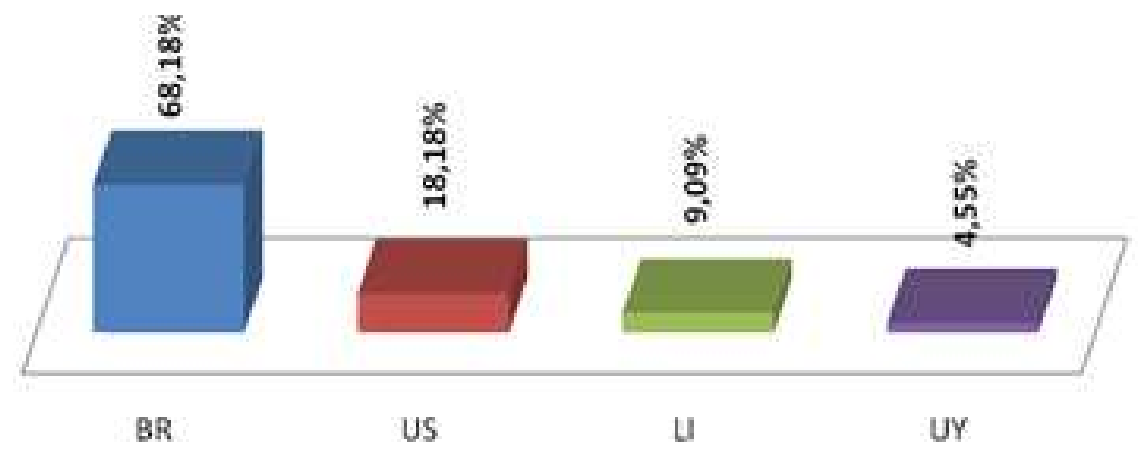

Fonte: Elaborado pelos autores deste artigo (2018) 
Na base de dados do ESPACENET, os países com maior número de depósitos ou prioridade são a China, com 40,91\%; e os Estados Unidos, com 21,59\% (Gráfico 3).

Gráfico 3 - Países de depósitos ou prioridade unionista - ESPACENET

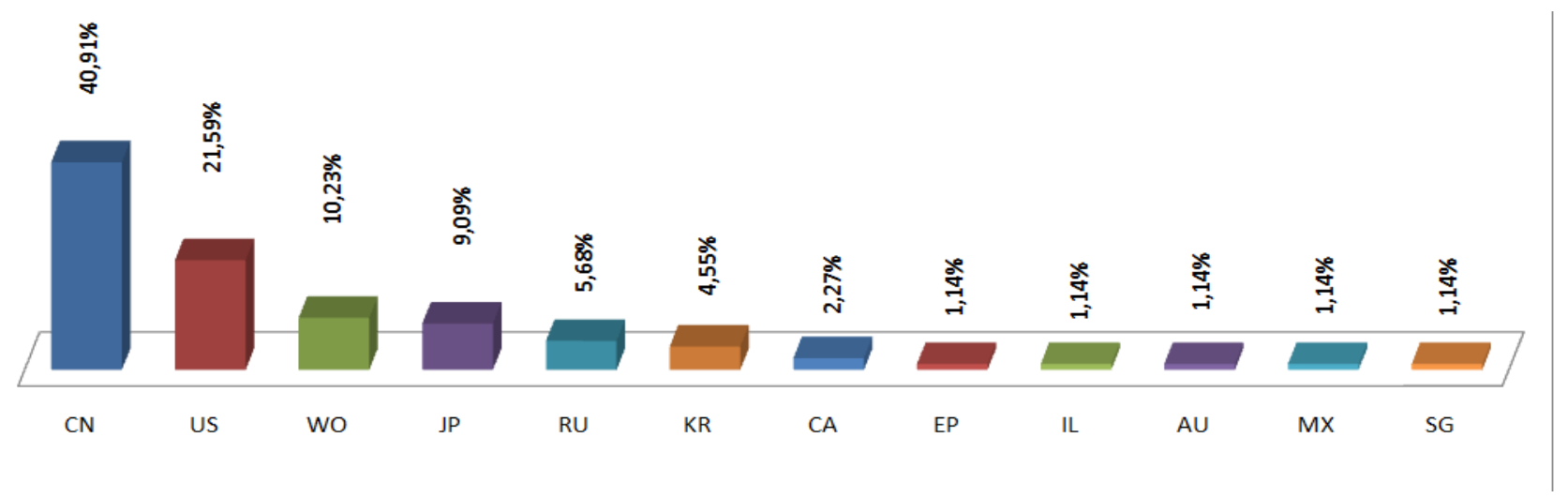

Fonte: Elaborado pelos autores deste artigo (2018)

Já na base do ORBIT ${ }^{\circledR}$, os principais países de depósitos ou prioridade são os Estados Unidos, com 8,11\%; e a Organização Europeia de Patentes, com 7,43\% (Gráfico 4).

Gráfico 4 - Países de depósitos ou prioridade unionista - ORBIT®

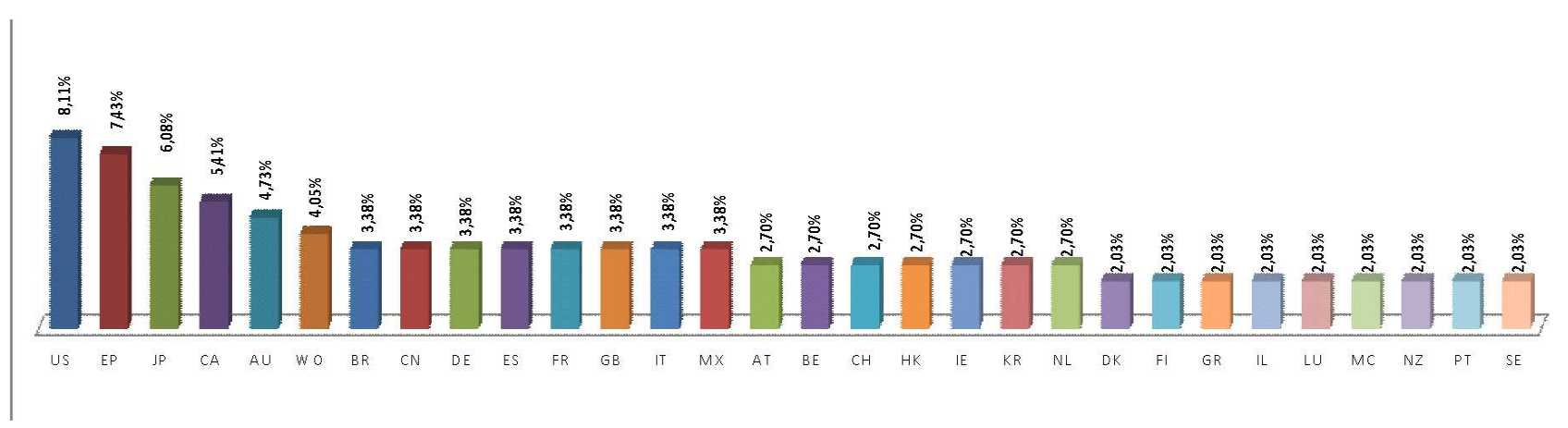

Fonte: Elaborado pelos autores deste artigo (2018)

Percebe-se que os Estados Unidos aparecem como o país de importância nos depósitos dos pedidos de patentes em todas as bases pesquisadas, liderando o número de depósitos no ORBIT®.

A situação do Brasil é bastante peculiar, pois, ao mesmo tempo em que empata com a China em quantidade de depósitos na base do ORBIT ${ }^{\circledR}$, não possui pedido de patente publicado na base do ESPACENET.

Com relação à pesquisa que mediu a quantidade de depósitos feitos, em cada uma das três bases, em um período de tempo preestabelecido, escolheu-se o período de 1998 a 2018 (20 anos) para análise (Gráfico 5). 
Gráfico 5 - Número de depósitos de pedidos de patente por ano (1998 a 2018)

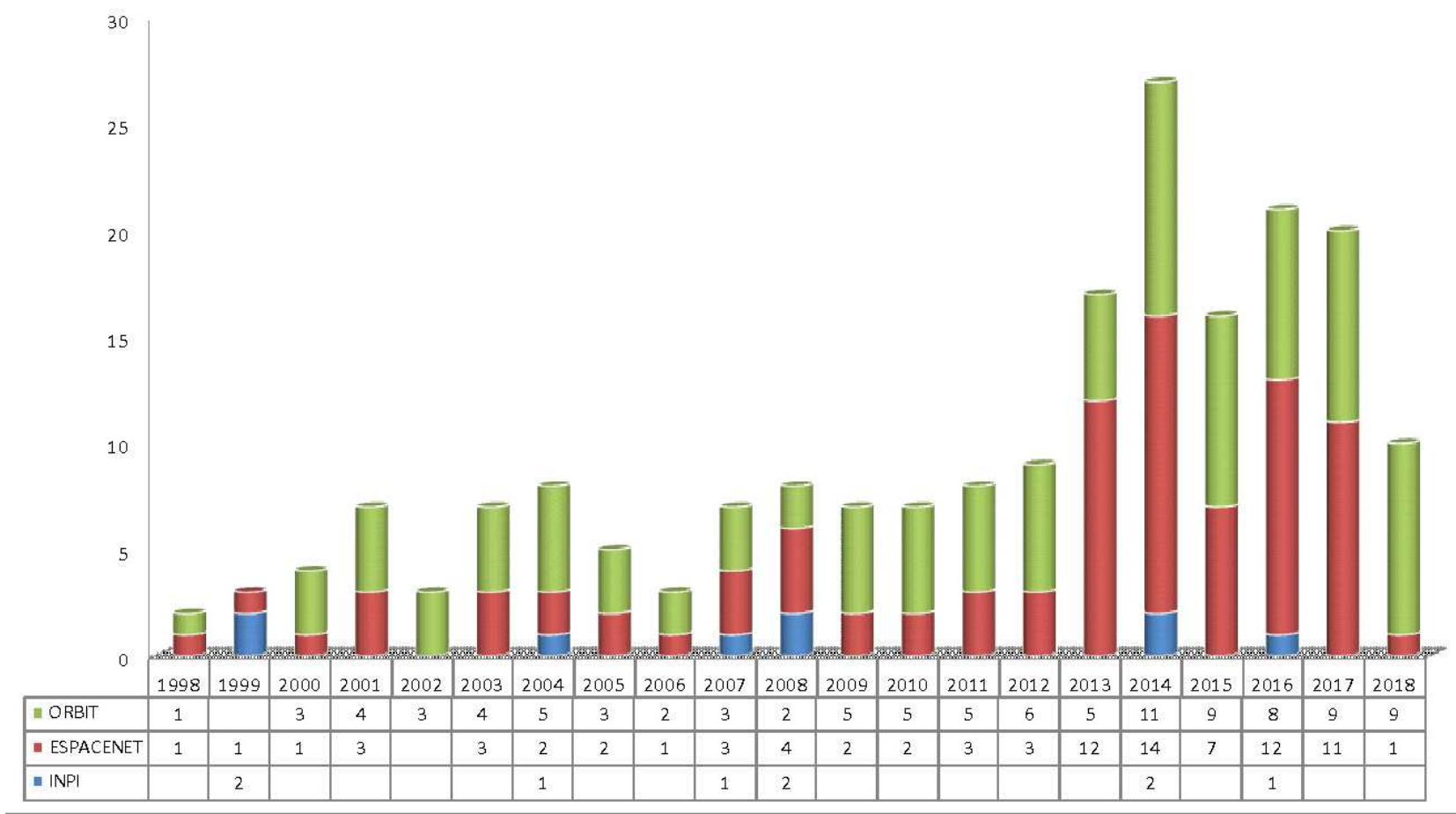

Fonte: Elaborado pelos autores deste artigo (2018)

Nota-se que houve uma regularidade nos depósitos a partir de 1998 na bases do ESPACENET e ORBIT ${ }^{\circledR}$, sendo que este último já possui nove patentes depositadas em 2018.

Com base no perfil do Gráfico 5, pode-se dizer que a evolução das tecnologias em estudo ao longo do tempo teve um crescimento moderado entre 1998 a 2012 e um crescimento alto entre 2013 a 2018, com destaque para o ano de 2014, ano em que houve um pico de interesse pelos depósitos de pedidos de patentes, sobretudo nas bases do ORBIT® e do ESPACENET.

A título de curiosidade, na base do INPI, o primeiro depósito com a temática da pesquisa foi realizado em 1992 e o último em 2016. No ESPACENET, o primeiro depósito ocorreu, ainda mais cedo, em 1983 e em 2018 houve um depósito. No ORBIT não foi possível obter informações sobre os depósitos de pedidos de patentes anteriores a 1998, sabe-se, porém, que em 2018 foram feitos nove depósitos.

A partir do mapeamento dos inventores nas bases pesquisadas percebe-se que Thorpe Philip e Mcghee William aparecem em todas elas com significativa relevância. No INPI, Renato Meneguelo e Marcos Vinicius são os maiores inventores, com três patentes inventadas cada um, seguidos por Mcghee William, Sophia Ran, Sandra Vasconcellos, Salvador Claro, Dr. Reinhard, Dr. Peter Hermann, Antônio José Reimann, Thorpe Philip, Peter Klein e Gilberto Orivaldo Chierice, todos com duas patentes inventadas (Gráfico 6). 
Gráfico 6 - Inventores - INPI

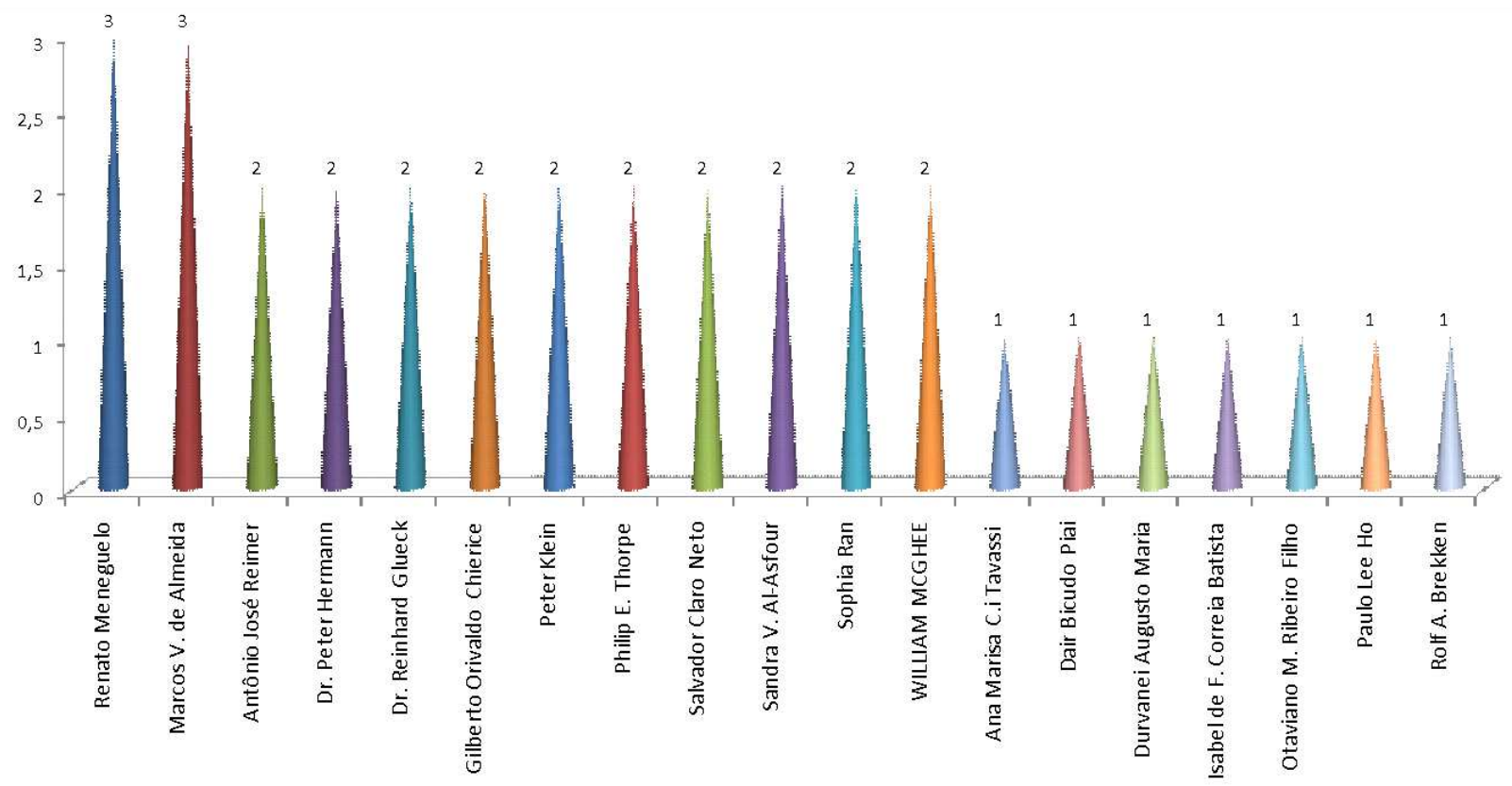

Fonte: Elaborado pelos autores deste artigo (2018)

Na base de dados do ESPACENET, os maiores inventores são Thorpe Philip, com 11 patentes inventadas; seguido por Mcghee William, Sophia Ran e Hejin, todos esses com cinco patentes inventadas (Gráfico 7).

Gráfico 7 - Inventores - ESPACENET

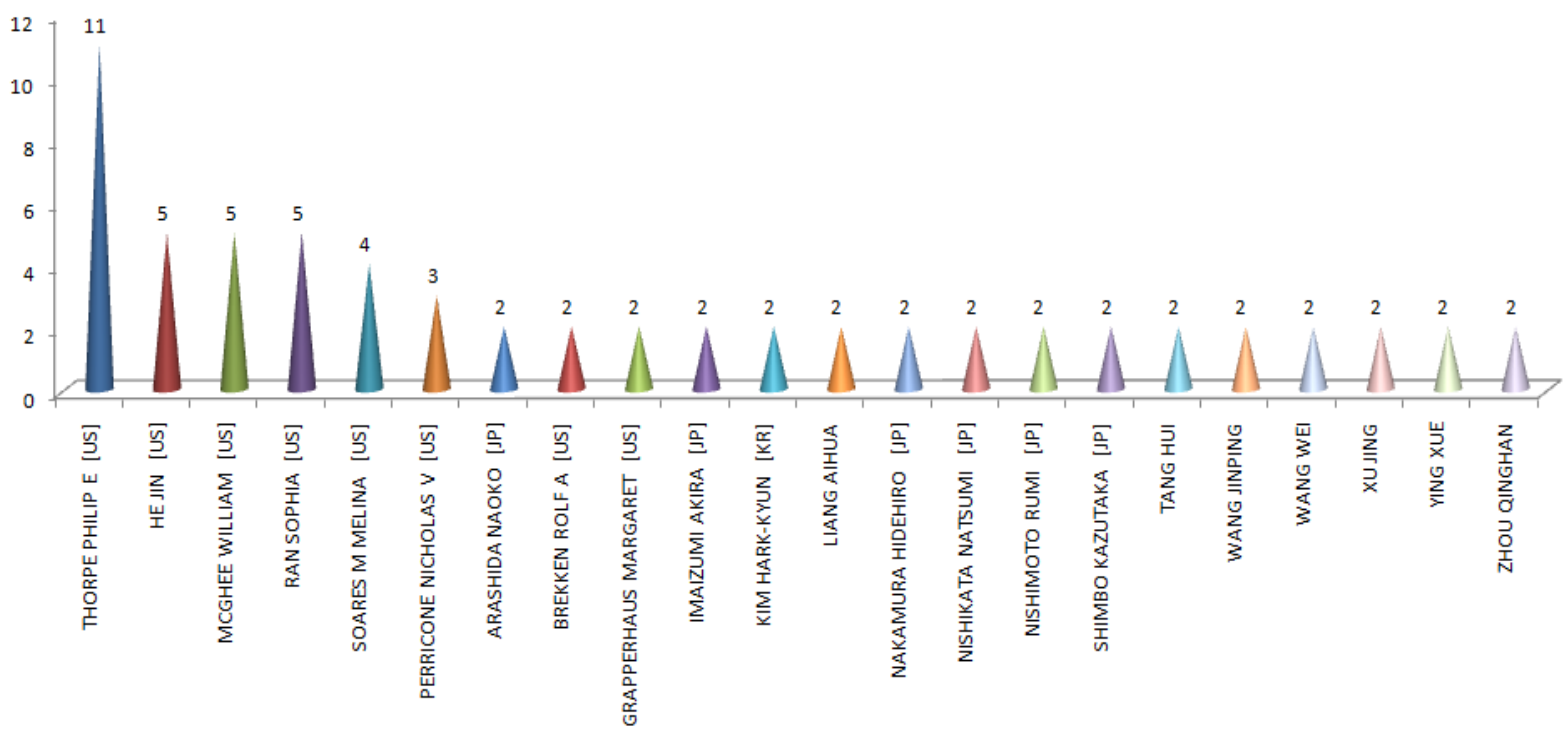

Fonte: Elaborado pelos autores deste artigo (2018) 
Já os três maiores inventores, segundo a base do ORBIT®, foram: Mcghee William, com cinco patentes inventadas; Thorpe Philip, com quatro patentes inventadas; e Sophia Ran, com três patentes inventadas (Gráfico 8).

Gráfico 8 - Inventores - ORBIT®

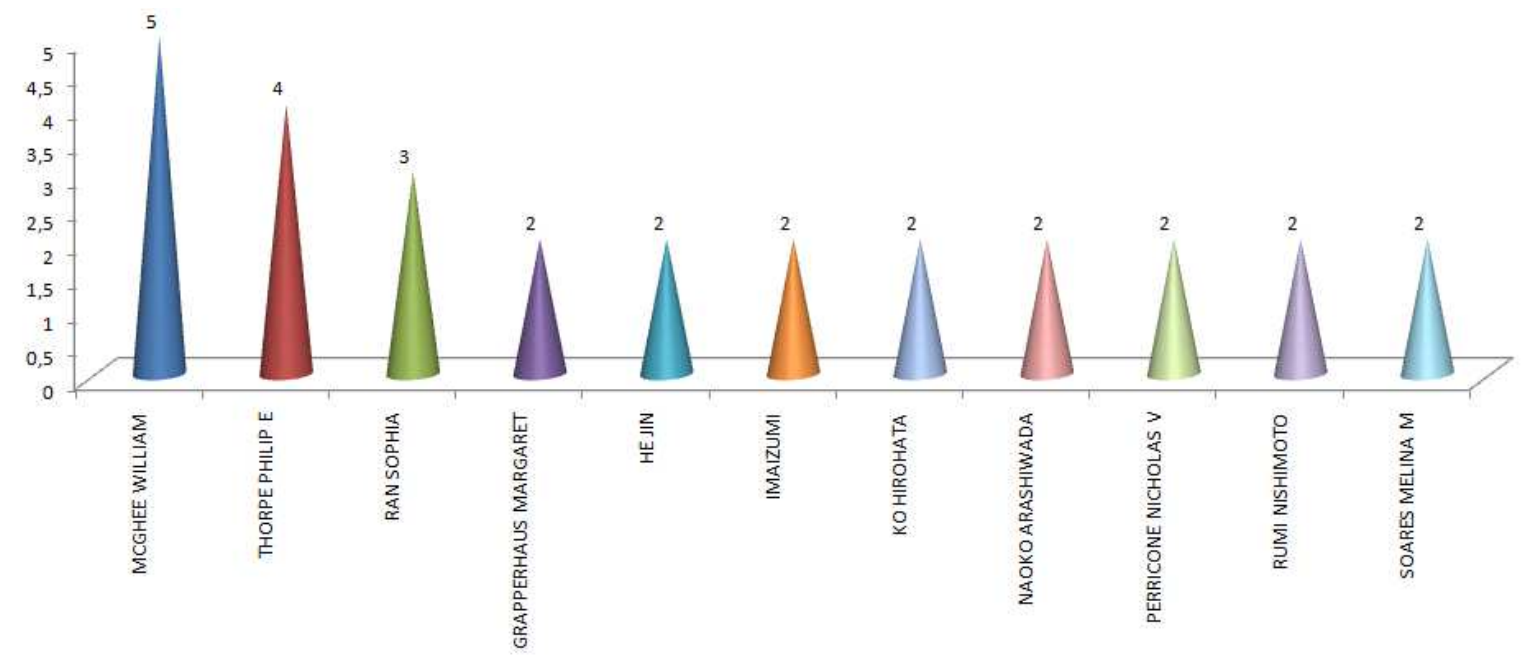

Fonte: Elaborado pelos autores deste artigo (2018)

Em síntese, observa-se que, no Brasil, os maiores inventores do tema são Renato Meneguelo e Marcos Vinicius, ambos com três patentes inventadas. No âmbito internacional, Thorpe Philip e Mcghee William são, respectivamente, os maiores inventores do tema.

Verifica-se que os maiores inventores são de nacionalidade americana, pois se destacam na quantidade absoluta de patentes inventadas.

Em relação aos depositantes, no INPI, os maiores depositantes da tecnologia pesquisada são Salvador Claro Neto, University of Texas; Sandra Vasconcellos, Renato Meneguelo, Nika Health Products Ltd.; Marcos Vinicius de Almeida, Mallinckrodt LCC; e Gilberto Chierice, todos são titulares de dois pedidos de patentes (Gráfico 9).

Gráfico 9 - Depositantes - INPI

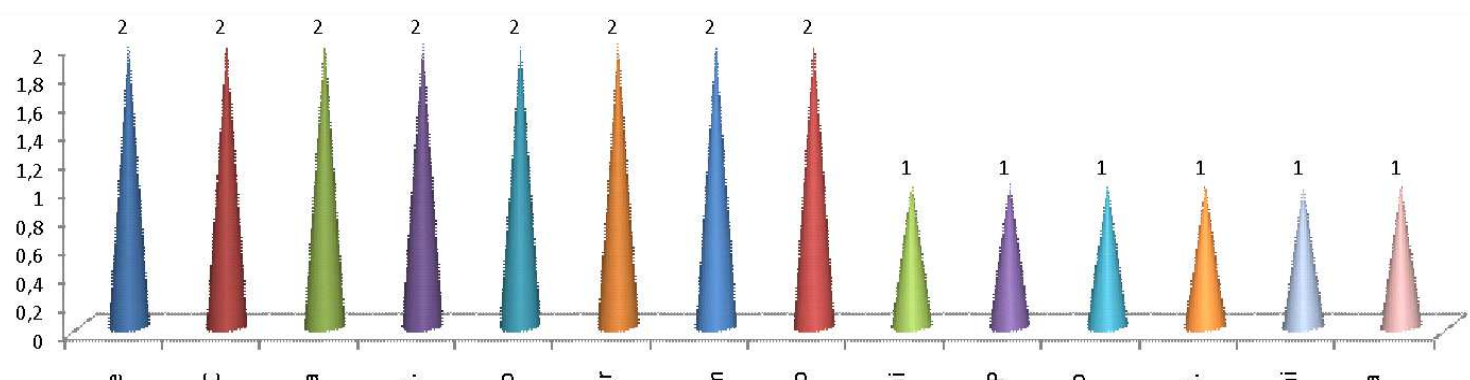

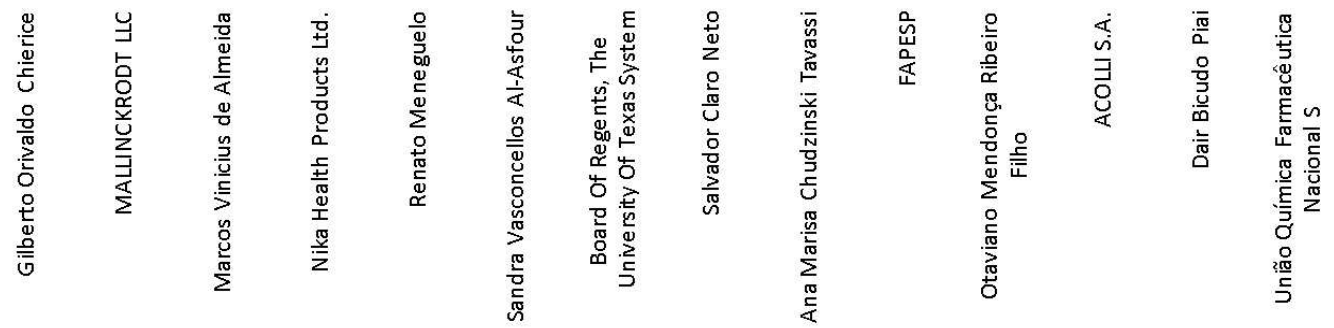

Fonte: Elaborado pelos autores deste artigo (2018) 
Já no ESPACENET, os maiores depositantes são: Chengdu Lvke Huatong Technology Co Ltd., titular de 13 pedidos de patentes; University of Texas, titular de 10 pedidos de patentes; Mallinckrodt LLC, titular de sete pedidos de patentes; Transdermal Biotechnology Inc., titular de três pedidos de patentes (Gráfico 10).

Gráfico 10 - Depositantes - ESPACENET

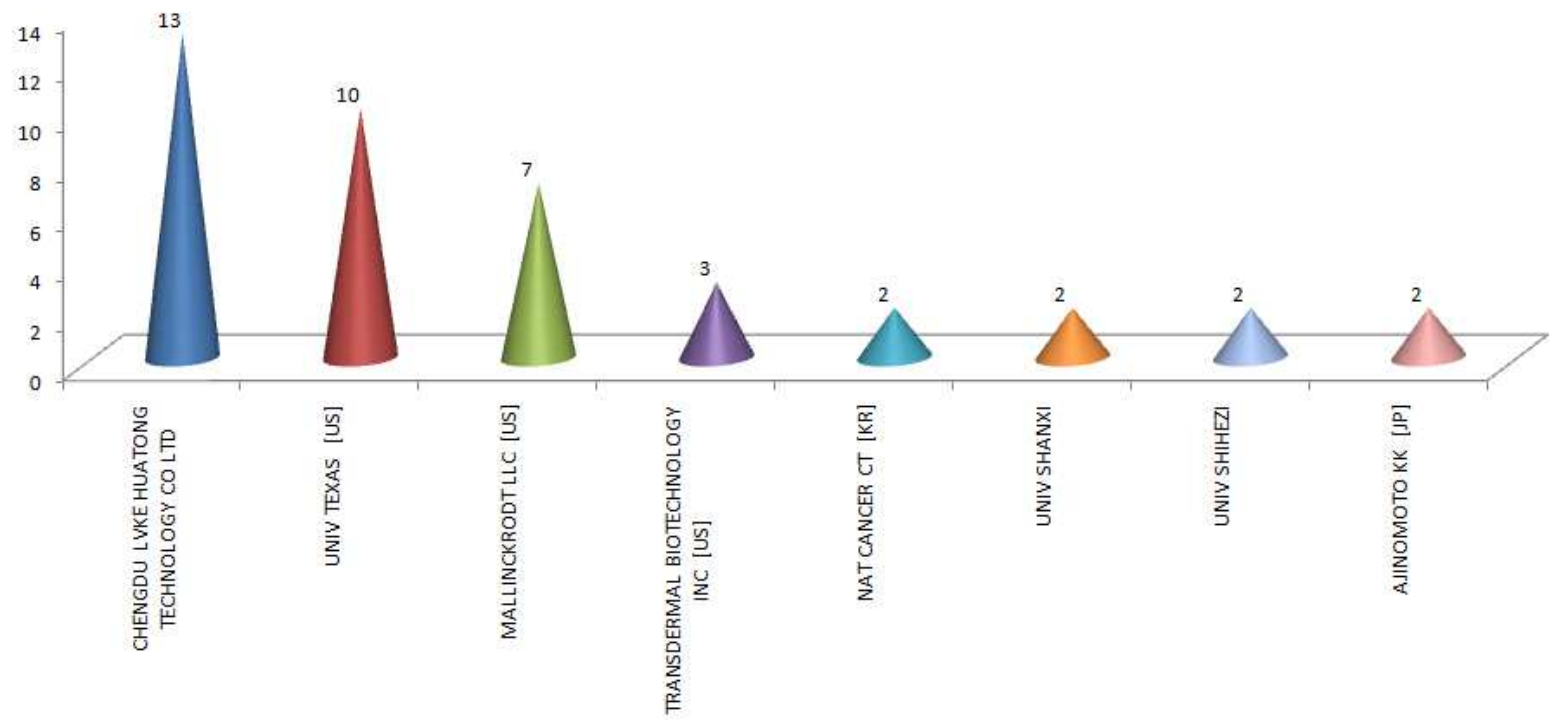

Fonte: Elaborado pelos autores deste artigo (2018)

No ORBIT®, os principais titulares são: Mallinckrodt LCC, com cinco pedidos de patentes; University of Texas, com quatro pedidos de patentes; Ajinomoto e Transdermal Biotechnology Inc., ambos com dois pedidos de patentes (Gráfico 11).

Gráfico 11 - Depositantes - ORBIT®

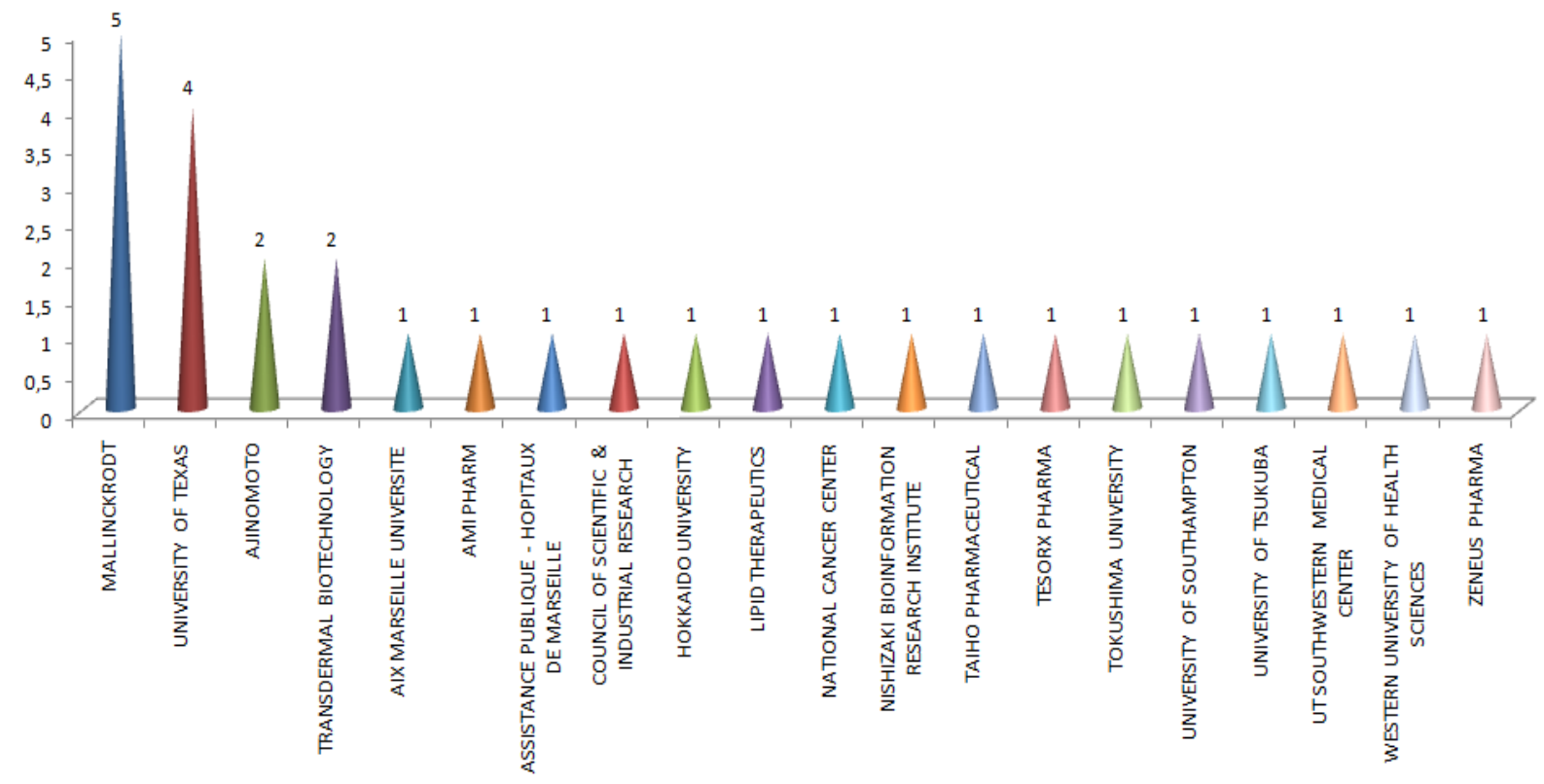

Fonte: Elaborado pelos autores deste artigo (2018) 
Nota-se que, no INPI, a quantidade de titulares pessoas físicas é maior do que a quantidade de titulares pessoas jurídicas, situação oposta ao que ocorre nas bases internacionais, ESPACENET e ORBIT ${ }^{\circ}$, em que a maioria dos depositantes são pessoas jurídicas, principalmente universidades e empresas.

A Universidade do Texas e a empresa Mallinckrodt LLC aparecem em todas as bases pesquisadas em posição de destaque, sendo que a Universidade do Texas teve 16 ocorrências de citações, e a Mallinckrodt LLC, 14 ocorrências de citações.

Todos os pedidos de patentes publicados são classificados na área tecnológica a que pertencem. Uma das classificações mais empregadas é a Classificação Internacional de Patentes (IPC), cujas áreas tecnológicas são divididas nas seções A a H. Dentro de cada seção, há classes, subclasses, grupos principais e subgrupos, com base em um sistema hierárquico. No gráfico 12 estão dispostos, por base de dados, os principais códigos IPCs consultados nesta pesquisa.

Gráfico 12 - Códigos de classificação internacional de patentes (IPC)

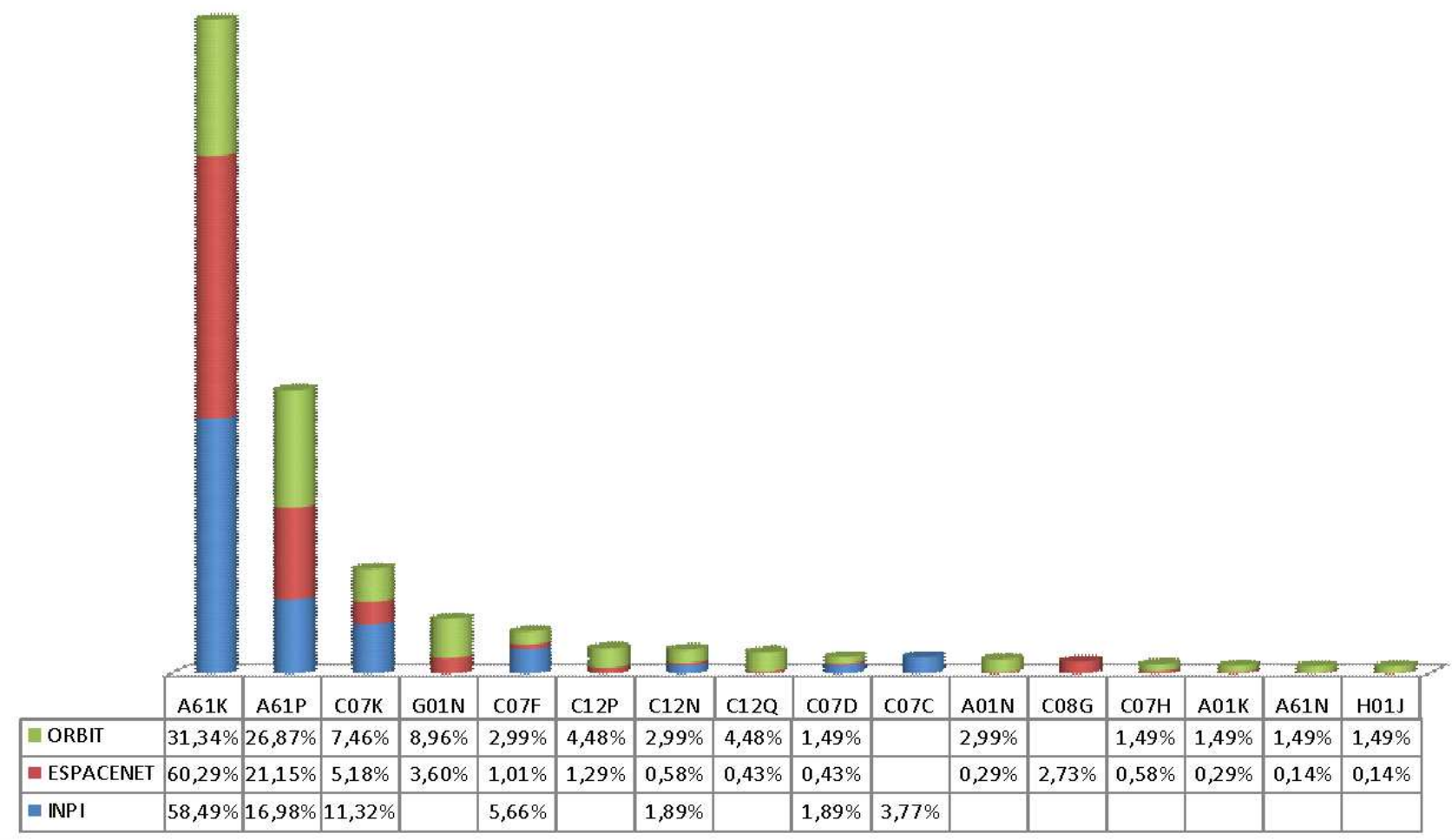

Fonte: Elaborado pelos autores deste artigo (2018)

Nas bases pesquisadas, a subclasse $\mathrm{A} 61 \mathrm{~K}$, que corresponde a preparações para finalidades médicas, odontológicas ou higiênicas (WIPO, 2018), retornou 58,49\% dos resultados no INPI, $60,29 \%$ no ESPACENET e 31,34\% no ORBIT®.

A subclasse A61P se refere à atividade terapêutica específica de compostos químicos ou preparações medicinais (WIPO, 2018) e teve um retorno de 16,98\% no INPI; $21,15 \%$ no ESPACENET; e $26,87 \%$ no ORBIT ${ }^{\circledR}$.

A subclasse C07K corresponde aos peptídeos (WIPO, 2018) e aparece em 11,32\% das patentes depositadas no INPI; $5,18 \%$ no ESPACENET; e 7,46\% no ORBIT®. 
Por fim, a subclasse G01N, que trata da investigação ou análise dos materiais pela determinação de suas propriedades químicas ou físicas (WIPO, 2018), não foi referenciada no INPI, mas aparece com 3,60\% das patentes depositadas no ESPACENET e com 8,96\% no ORBIT ${ }^{\circledR}$.

As subclasses A61K e A61P foram as que apresentaram, respectivamente, a maior incidência nas bases pesquisadas, evidenciando a aplicação da fosfoetanolamina nas preparações medicinais e a atividade terapêutica específica desse composto químico.

\section{Considerações Finais}

Com base nos dados de depósitos de patentes pesquisados, verificou-se que a manipulação da fosfoetanolamina, com o objetivo de diagnóstico, prevenção ou tratamento do câncer, já vem sendo documentada na forma de patente desde 1983, ou seja, não é algo tão recente.

Os Estados Unidos é o país que está na vanguarda em relação ao tema, com maior número total de depósitos de pedidos de patentes e é o país de origem da maioria dos inventores.

A maioria dos depositantes é de pessoas jurídicas e, em alguns casos, o depositante é também o inventor da patente.

A Universidade do Texas é a maior titular de pedidos de patentes sobre a fosfoetanolamina associada ao diagnóstico, à prevenção ou ao tratamento de algum tipo de câncer.

Quanto à área tecnológica dos documentos de patentes recuperados, verificou-se que, em relação à classificação internacional de patentes, a subclasse A61K e A61P, ambas da seção A (Necessidades Humanas), Subclasse A61 (Ciência Médica ou Veterinária; Higiene), tiveram preponderância nos resultados, o que já era esperado devido à estratégia de busca adotada nas bases escolhidas.

Essa subclasse (A61K) abrange o seguinte assunto, seja estabelecido como uma composição (mistura), processo de preparação da composição ou processo de tratamento usando a composição:

Droga ou outras composições biológicas que são capazes de:

a) prevenir, aliviar, tratar ou curar condições anormais ou patológicas do corpo vivo por meios de como destruir um organismo parasita, ou limitar o efeito da doença ou anormalidade alterando quimicamente a fisiologia do hospedeiro ou parasita;

b) manutenção, aumento, diminuição, limitação ou destruição de uma função fisiológica do corpo, por exemplo, composições vitamínicas, esterilizantes sexuais, inibidores de fertilidade, promotores de crescimento ou similares; $e$

c) diagnosticar uma condição ou estado fisiológico por um teste in vivo, por exemplo, contraste de raios $\mathrm{X}$ ou composições de teste de adesivos cutâneos.

A atividade terapêutica de preparações medicinais é ainda classificada na subclasse A61P. 


\section{Referências}

EPO - European Patent Office. [2018]. Disponível em: https://worldwide.espacenet.com/ advancedSearch?locale =en_EP. Acesso em: 25 set. 2018.

GUERREIRO, E. S. et al. Análise de documentos de patentes sobre copaíba: uma comparação entre fontes de dados. Cadernos de Prospecção, Salvador, v. 11, n. 1, p. 26-40, jan./mar., 2018.

INPI - INSTITUTO NACIONAL DA PROPRIEDADE INDUSTRIAL. Consulta à base de dados do INPI. [2016]. Disponível em: https://gru.inpi.gov.br/pePI/servlet/LoginController?action=login. Acesso em: 25 set. 2018.

INPI - INSTITUTO NACIONAL DA PROPRIEDADE INDUSTRIAL. Informação tecnológica: Busca de patentes. [2018]. Disponível em http://www.inpi.gov.br/menu-servicos/informacao/busca-depatentes. Acesso em: $26 \mathrm{dez} .2018$.

ORSI, Carlos. Fosfoetanolamina, o "caso que envergonhou a ciência brasileira": como o populismo aliado à ignorância científica produziu um dos piores momentos da ciência nacional em décadas. Gazeta do Povo, Seção Medicina, Curitiba, 2017. Disponível em: https://www. gazetadopovo.com.br/ideias/fosfoetanolamina-ocaso-que-envergonhou-a-ciencia-brasileirad5wnxh6h28oop2z9b3xsg6v3w/. Acesso em: 20 set. 2018.

PIVETTA, Marcos. A prova final da fosfoetanolamina: Pesquisa FAPESP 243. São Paulo, 2016. p. $17-23$.

PROFNIT - MESTRADO PROFISSIONAL EM PROPRIEDADE INTELECTUAL E TRANSFERÊNCIA DE TECNOLOGIA PARA INOVAÇÃO. [2018]. Disponível em: http://www.profnit.org.br/pt/samplepage/. Acesso em: 20 set. 2018.

QUESTEL Co. Orbit at a glance. [2018]. Disponível em: https://www.questel.com/software/orbit-ata-glance/. Acesso em: 25 set. 2018.

SOUZA, M. A. de; PRADO, F. O. do. Operacionalização da propriedade industrial. In: PORTO, G. S. Gestão da Inovação e Empreendedorismo. 1. ed. Rio de Janeiro: Elsevier, 2013. p. 171-203.

STF - SUPREMO TRIBUNAL FEDERAL (Brasil). STF suspende eficácia da lei que autoriza uso da fosfoetanolamina. Seção Notícias STP. São Paulo, 2016. Disponível em: http://www.stf.jus.br/ portal/cms/verNoticiaDetalhe.asp?idConteudo=317011. Acesso em: 20 set. 2018.

SUZUKI, Henry. Orbit Intelligence: Visão Geral Sobre o Sistema. 2017. (Slides). Disponível em: https://axonal.com.br/arquivos/PDF/Orbit_Visao_Geral_Sistema_PARTES_1_a_3_BUSCA_ VISUALIZACAO_SELECAO.pdf. Acesso em: $\overline{2} 6 \mathrm{dez} .2018$.

WIPO - WORLD INTELLECTUAL PROPERTY ORGANIZATION. Classificação Internacional de Patentes (IPC). [2018]. Disponível em: http://www.wipo.int/classifications/ipc/en/. Acesso em: 28 set. 2018. 


\section{Sobre os Autores}

\section{Tarcísio Magalhães Cerqueira}

E-mail: tarcisio.nit@gmail.com

Bacharel em Direito.

Endereço profissional: Universidade Estadual de Feira de Santana, Reitoria, Pró-Reitoria de Pesquisa e PósGraduação. Avenida Transnordestina, s/n, Novo Horizonte, Feira de Santana, BA. CEP: 44036-900.

\section{Marcelo Miguel da Silva}

E-mail:mmiguel.engmec@gmail.com

Engenheira Mecânica.

Endereço profissional: Instituto Federal da Bahia, Instituto Federal de Educação, Ciência e Tecnologia da Bahia. Via Universitária, s/n, Pintaguinha, Simões Filho, BA. CEP: 43700-000.

\section{Nubia Moura Ribeiro}

E-mail: nubiamouraribeiro@gmail.com

Engenharia Química.

Endereço profissional: Instituto Federal da Bahia, Campus Jequié. John Kennedy, s/n Loteamento, Cidade Nova, Jequié, BA. CEP: 45201-570. 